\title{
Imitation of ultra-sharp light focusing within turbid tissue-like scattering medium by using time-independent Helmholtz equation and method Monte Carlo
}

Buligin, A., Kistenev, Y., Meglinski, I., Danilkin, E., Vrazhnov, D.

A. D. Buligin, Y. V. Kistenev, I. Meglinski, E. A. Danilkin, D. A. Vrazhnov, "Imitation of ultra-sharp light focusing within turbid tissue-like scattering medium by using time-independent Helmholtz equation and method Monte Carlo," Proc. SPIE 11582, Fourth International Conference on Terahertz and Microwave Radiation: Generation, Detection, and Applications, 115821N (17 November 2020); doi: 10.1117/12.2582667

Event: Fourth International Conference on Terahertz and Microwave Radiation: Generation, Detection, and Applications, 2020, Tomsk, Russian Federation 


\title{
Imitation of ultra-sharp light focusing within turbid tissue-like scattering medium by using time-independent Helmholtz equation and method Monte Carlo
}

\author{
Bulygin A.D. ${ }^{1,2, *}$, Kistenev Y.V. ${ }^{2}$, Meglinski I. ${ }^{2,3,4,5}$, Danilkin E.A. ${ }^{1,2}$, Vrazhnov D.A. ${ }^{2,6}$ \\ ${ }^{1}$ Federal state V.E. Zuev Institute of Atmospheric Optics of Siberian Branch of the Russian \\ Academy of Science, Tomsk, Russia \\ 2 Tomsk State University, Laboratory of Biophotonics, Tomsk, Russia \\ ${ }^{3}$ University of Oulu, Optoelectronics and Measurement Techniques Laboratory, Oulu, Finland \\ ${ }^{4}$ Aston University, School of Engineering and Applied Science, Birmingham, UK \\ ${ }^{5}$ Aston University, School of Life and Health Sciences, Birmingham, UK \\ ${ }^{6}$ Institute of Strength Physics and Materials Science of SB RAS, Tomsk, Russia
}

\begin{abstract}
Based on time-independent Helmholtz equation and its solution in frame of inhomogeneous approximation a hybrid computational method for imitation of propagation of bounded laser beam focused into biological tissue is introduced. The biological tissue is simulated as a semi-infinite randomly inhomogeneous medium. The developed approach is intended to model laser beams in the super-sharp focusing mode. The results of modeling of laser light focusing into the turbid tissue-like scattering medium with lenses of various shapes are presented.
\end{abstract}

Keywords: Helmholtz equation, biotissue, Monte Carlo method, light scattering model

\section{INTRODUCTION}

Nowadays the imaging of biological tissues utilizing optical coherence tomography (OCT) is widely in use [1-5]. The variation of the depth of visualization and spatial resolution of the technique can be achieved utilizing focusing lenses. In this case, it is necessary to take into account the transverse structure of the probing optical radiation. Typically, the bounded optical beams with a Gaussian transverse profile are used [1-3, 5].

The properties of the light field in the focus of the beam are determined by the shape and parameters of the lens, e.g., a spherical lens studied in [6-8]. An analysis of the focusing of light beam into a scattering medium by spherical lenses in can be performed analytically in some cases [8]. However, the study of bounded focusing beams in a randomly inhomogeneous medium, for example, biological tissue, requires the use of numerical methods.

A numerical approach based on the paraxial approximation has been developed [9-13], but its use becomes illconditioned for lenses with a numerical aperture value greater than or equal to one [6-8].

An approach to modeling the propagation of optical radiation within biological tissues based on the corpuscular Monte Carlo method is well known [1-3], has been applied for the study of OCT image formation [14], and later modified for the modeling of polarization-based OCT $[15,16]$. In fact, this approach has several limitations [17] that manifest itself in super-sharp focusing when the numerical aperture is close to or less than one.

* Correspondence: b.a.d@iao.ru;

Fourth International Conference on Terahertz and Microwave Radiation: Generation, Detection, and Applications, edited by Oleg A. Romanovskii, Yurii V. Kistenev, Proc. of SPIE Vol. 11582

$115821 \mathrm{~N} \cdot$ @ $2020 \mathrm{SPIE} \cdot \mathrm{CCC}$ code: $0277-786 \mathrm{X} / 20 / \$ 21 \cdot$ doi: $10.1117 / 12.2582667$ 
Current study aims to develop numerical methods to mimic focusing bounded light beams and its propagation in a randomly inhomogeneous medium with lenses of arbitrary shape, including ultra-sharp focusing by both wave and corpuscular Monte Carlo methods.

\section{MATHEMATICAL MODEL OF THE PROPAGATION PROBLEM}

To study the focusing of the bounded light beams in a randomly inhomogeneous medium by lenses of arbitrary shape, we use the time-independent Helmholtz equation:

$$
\Delta \psi(r)+k_{0}^{2} \psi(r)=0
$$

Here, $\psi$ is the complex amplitude of the field strength of the light wave $E(\boldsymbol{r}, t)$, which corresponds to the representation of the field in the form $E(\boldsymbol{r}, t)=\psi(\boldsymbol{r}) e^{-i \omega_{0} t}$. The wavenumber $k_{0}$ is expressed through the angular frequency $\omega_{0}$ as follows:

$$
k_{0}=\omega_{0} / c .
$$

Choosing one of the $z$ coordinates and rewriting (1) in the form

$$
\partial_{z}^{2} \psi\left(z, \mathbf{r}_{\perp}\right)=-\left(\Delta_{\perp}+k_{0}^{2}\right) \psi\left(z, \mathbf{r}_{\perp}\right),
$$

where $\mathbf{r}_{\perp}$ is the radius vector in a plane transverse to the direction of propagation along $z$; the transverse Laplacian has the form

$$
\Delta_{\perp}=\partial_{x}^{2}+\partial_{y}^{2}
$$

Using the Fourier transform, we represent the required field in the following form

$$
\psi\left(z, \mathbf{r}_{\perp}\right)=\int_{R^{3}} \tilde{\psi}\left(k_{z}, \mathbf{k}_{\perp}\right) e^{-i\left(k_{z} z-k_{z} \mathbf{r}_{\perp}\right)} d k_{z} d \mathbf{k}_{\perp} .
$$

Next, we introduce the assumption that the Fourier transform of the field is localized in the region $k_{z}>0$, and

$$
\left|\mathbf{k}_{\perp}\right|<k_{0} .
$$

We consider the unidirectional Helmholtz equation in a randomly inhomogeneous medium for modeling biological tissue similarly to [15]. In this case, the equation for the field $\psi$ will have the form

$$
\partial_{z}^{2} \psi\left(z, \mathbf{r}_{\perp}\right)=-\left(\Delta_{\perp}+k_{0}{ }^{2}\left(\left\langle n\left(z, \mathbf{r}_{\perp}\right)\right\rangle^{2}+2\left\langle n\left(z, \mathbf{r}_{\perp}\right)\right\rangle \delta \tilde{n}\left(z, \mathbf{r}_{\perp}\right)\right)\right) \psi\left(z, \mathbf{r}_{\perp}\right) .
$$

where $n=\langle n\rangle+\delta \tilde{n}$ defines random variations of the refractive index of the medium, with a separated regular and fluctuation part, respectively. The random Gaussian field of fluctuations of the medium is constructed following the model in [5, 15], where for the correlation function of the field $\delta \tilde{n}$ following relation is held

$$
\langle\delta \tilde{n}(\mathbf{r}) \delta \tilde{n}(\mathbf{r}+\delta \mathbf{r})\rangle=\int_{R^{3}} \frac{C_{0}}{1+\left(l_{c} \mathbf{k}\right)^{2 D_{f}}} e^{-i \mathbf{k r}} d \mathbf{k},
$$

where $l_{c}$ is the correlation radius, $D_{f}$ is the refractive index of the fluctuation of roughness parameter, $C_{0}$ is the characteristic of the dispersion of fluctuations of the refractive index. The defined set of these parameters determines the type of biological tissue [15]. These parameters can be correlated with the parameters of the indicatrix of the medium [5] used in the corpuscular Monte Carlo method for modeling the propagation of radiation in biological tissue. 


\section{NUMERICAL MODELING}

To solve equation (4), the method of splitting in physical parameters was used, similar to [5]. At the diffraction propagation step, equation (2) is solved, which has the following form

$$
\partial_{z}^{2} \tilde{\psi}\left(z, \mathbf{k}_{\perp}\right)=\left(-\mathbf{k}_{\perp}^{2}+k_{0}^{2}\right) \tilde{\psi}\left(z, \mathbf{k}_{\perp}\right) .
$$

Then, the expression for transforming the field upon passing a small step along the evolutionary variable is

$$
\tilde{\psi}\left(z+\delta z, \mathbf{r}_{\perp}\right)=\int_{\mathbf{k}_{\perp} \in \Omega} d \mathbf{k}_{\perp} e^{-i \mathbf{k}_{\perp} \mathbf{r}_{\perp}} \tilde{\psi}\left(z, \mathbf{k}_{\perp}\right) e^{-i \delta z \sqrt{k_{0}^{2}-\mathbf{k}_{\perp}{ }^{2}}} .
$$

The phase incursion when passing a step in a randomly inhomogeneous medium is given by the following relation

$$
\tilde{\psi}\left(z+\delta z, \mathbf{r}_{\perp}\right)=\tilde{\psi}\left(z, \mathbf{r}_{\perp}\right) e^{-i k_{0}^{2} \Delta \tilde{\delta} \delta z},
$$

where $\Delta \tilde{n}=\left(\left\langle n\left(z, \mathbf{r}_{\perp}\right)\right\rangle^{2}+2\left\langle n\left(z, \mathbf{r}_{\perp}\right)\right\rangle \delta \tilde{n}\left(z, \mathbf{r}_{\perp}\right)\right)-1$.

Thus, we represent the step of passing in the form:

$$
\tilde{\psi}\left(z+\delta z, \mathbf{r}_{\perp}\right)=\left[\int_{\mathbf{k}_{\perp} \in \Omega} d \mathbf{k}_{\perp} e^{-i \mathbf{k}_{\perp} \mathbf{r}_{\perp}} \tilde{\psi}\left(z, \mathbf{k}_{\perp}\right) e^{-i \delta z \sqrt{k_{0}^{2}-\mathbf{k}_{\perp}{ }^{2}}}\right] e^{-i k_{0}^{2} \Delta \tilde{n} \delta z} .
$$

In the numerical implementation, the Fast Fourier transform from the parallel version of the MKL library was used. The medium model was chosen with the following parameters: $n=1.54, l_{c}=1.5 \mu m, D_{f}=3.9$. The initial beam profile was set in the Gaussian form

$$
\psi\left(z, \mathbf{r}_{\perp}\right)=e^{-\frac{\mathbf{r}_{\perp}{ }^{2}}{2 r_{0}^{2}}} e^{-i \phi\left(\mathbf{r}_{\perp}\right)},
$$

where the beam size $r_{0}$ was chosen equal to $0.6 \mathrm{~mm}$ and the characteristic scale of the radius of curvature of the phase front $\varphi$ was of the order of $1 \mathrm{~mm}$. Focusing with a spherical parabolic lens and a fraxicon lens was considered as an example. In this case, the phase has the following form, respectively:

$$
\begin{gathered}
\phi\left(r_{\perp}\right)=\frac{k_{0} r_{\perp}^{2}}{2 f} \\
\varphi\left(r_{\perp}\right)=1-\sqrt{\left|1-2\left(r_{\perp} / f\right)^{2}\right|} \\
\phi\left(r_{\perp}\right)=\left\{\begin{array}{l}
\frac{k_{0} r_{\perp}^{2}}{2 f} \text { if } r_{\perp} / f<1 \\
\frac{k_{0} r_{\perp}}{2} \text { if } r_{\perp} / f>1
\end{array}\right.
\end{gathered}
$$

Below, in Figures 1-3, the results of modeling the propagation of a light beam during focusing are presented. 


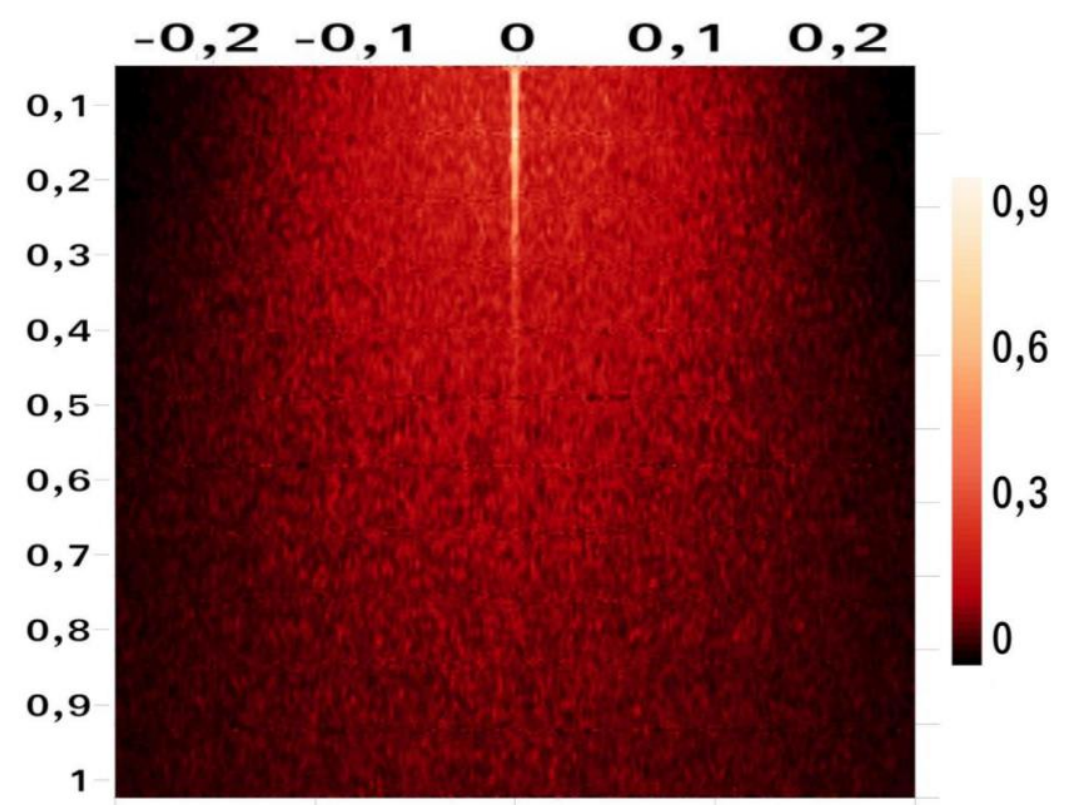

Figure 1. The results of modeling the propagation of a light beam during focusing with phase in the form (8).

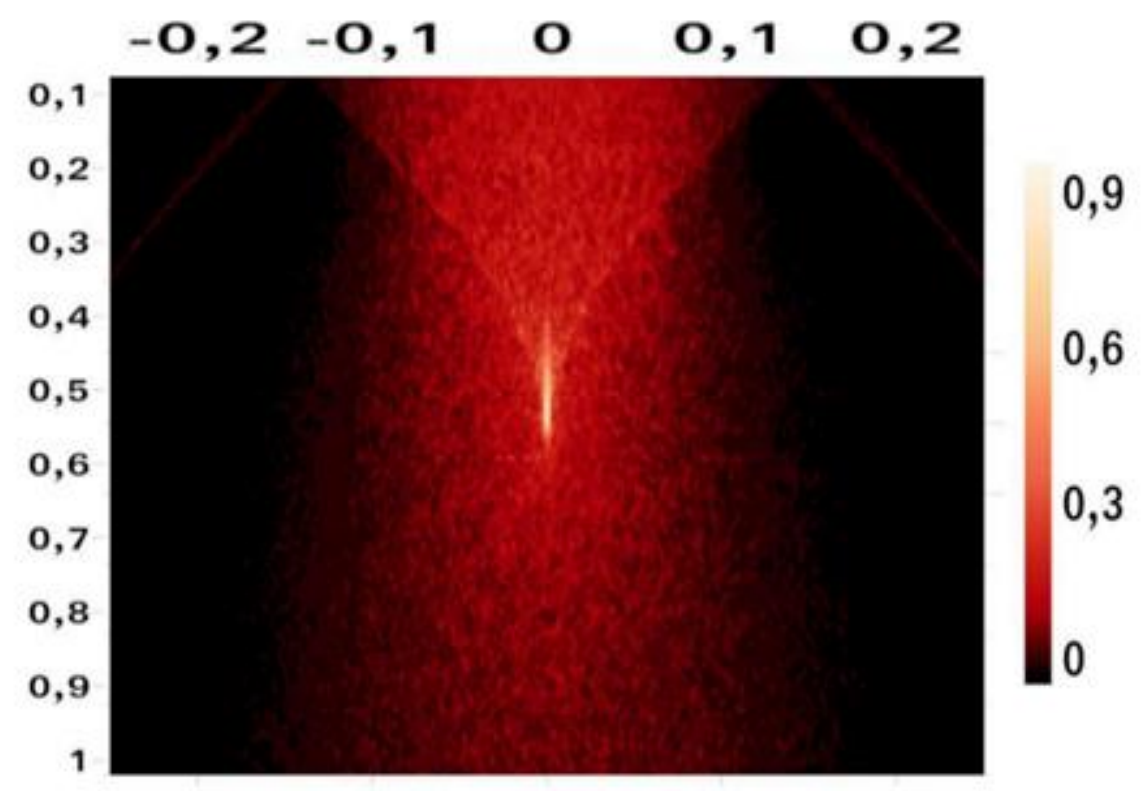

Figure 2. The results of modeling the propagation of a light beam during focusing with phase in the form (9). 


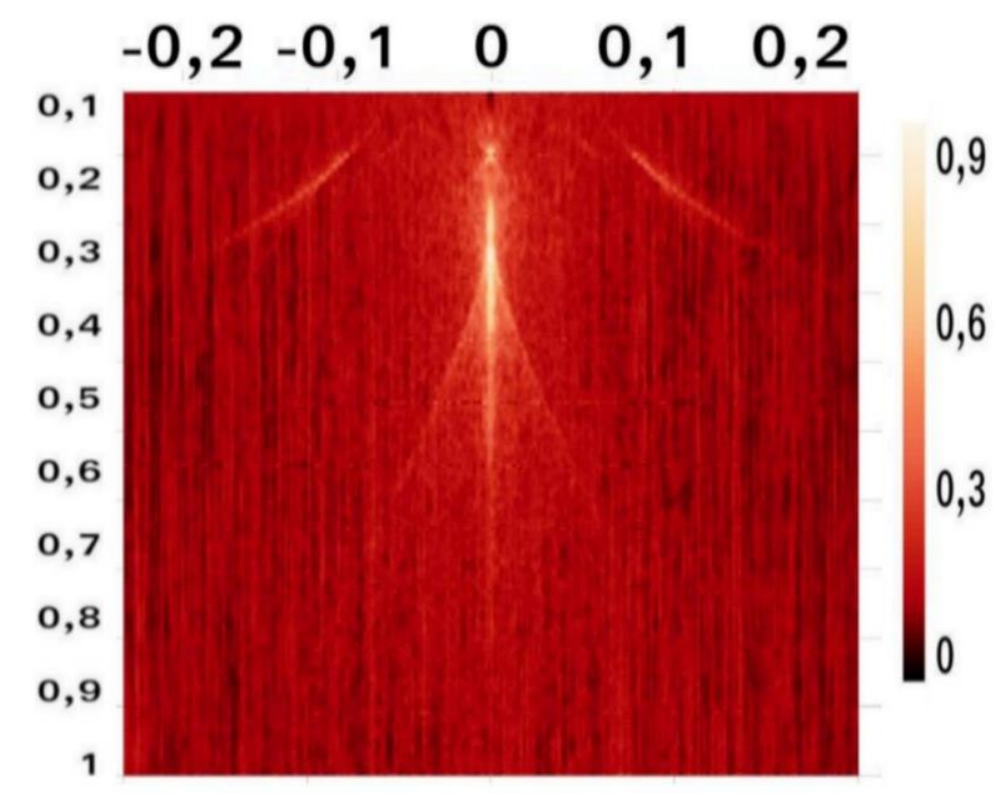

Figure 3. The results of modeling the propagation of a light beam during focusing with phase in the form (10).

The difference in the presented pictures is due to the distribution of the field at the focus of the lenses. The field is mostly localized when focusing on a conventional parabolic lens.

\section{DISCUSSION}

In this work, a numerical methodology for studying the propagation of laser radiation in biological tissue was developed based on the unidirectional Helmholtz equation. An example of a numerical calculation of the propagation of laser radiation in biological tissue for the case of focusing radiation with lenses of various shapes is given. The proposed numerical methodology can be used to simulate the visualization of biological tissue by optical coherence tomography for optimization purposes.

\section{ACKNOWLEDGMENTS}

The work was performed under the government statement of work for ISPMS Project No. III.23.2.10.

The reported study was partially funded by RFBR and Tomsk region according to the research project № 18-42-703012. The reported study was partially funded by RFBR and Tomsk region according to the research project № 19-42-703015. The reported study was partially funded by the Russian Fund of Basic Research (grant No.17 - 00 - 00186).

\section{REFERENCES}

[1] Matveyev A. L., Matveev L. A., Moiseev A. A., Sovetsky A. A., Gelikonov G. V., Zaitsev V. Y. "Semianalytical full-wave model for simulations of scans in optical coherence tomography with accounting for beam focusing and the motion of scatterers," Laser Phys. Lett., 16(8), 085601 (2019).

[2] Kalkman J. "Fourier-domain optical coherence tomography signal analysis and numerical modeling," International Journal of Optics, 2017, 9586067 (2017). 
[3] Jensen M., Israelsen N.M., Maria M., Feuchter T., Podoleanu A., Bang O. "All-depth dispersion cancellation in spectral domain optical coherence tomography using numerical intensity correlations," Sci. Rep., 8(1), 1-10 (2018).

[4] Meglinski I., Kirillin M., Kuzmin V. Myllylä R. "Simulation of polarization-sensitive optical coherence tomography images by a Monte Carlo method," Opt. Lett. 33(14), 1581-1583 (2008).

[5] Bulygin A., Vrazhnov D., Sim E., Meglinski I., Kistenev Yu. "Imitation of optical coherence tomography images by wave Monte Carlo-based approach implemented with the Leontovich-Fock equation,” Opt. Eng. 59(6), 061626 (2020), doi: 10.1117/1.OE.59.6.061626

[6] Schmitt J.M., Knüttel A. "Model of optical coherence tomography of heterogeneous tissue," J. Opt. Soc. Am. A. 14(6), 1231-42 (1997).

[7] Maxwell J.C. The Scientific Papers. v.1, Cambr. Univ.Press (1890).

[8] Kotlyar V.V., Melekhin A.S. "Abel transform in problems of synthesis of gradient optical elements," Computer Optics 24, 53-57 (2002).

[9] Pan Y., Birngruber R., Rosperich J., Engelhardt R. "Low-coherence optical tomography in turbid tissue: theoretical analysis," Appl. Opt., 34(28), 6564-6574 (1995).

[10] Smithies D.J., Lindmo T., Chen Z., Nelson J.S., Milner T.E. "Signal attenuation and localization in optical coherence tomography studied by Monte Carlo simulation," Phys. Med. Biol., 43(10), 3025 (1998).

[11] Yao G., Wang L.V. "Monte Carlo simulation of an optical coherence tomography signal in homogeneous turbid media," Phys. Med. Biol., 44(9), 2307 (1999).

[12] Kirillin M.Y., Farhat G., Sergeeva E.A., Kolios M.C., Vitkin A. "Speckle statistics in OCT images: Monte Carlo simulations and experimental studies," Opt. Lett., 39(12), 3472-3475 (2014).

[13] Hokr B.H., Bixler J.N., Elpers G., Zollars B., Thomas R.J., Yakovlev V.V., Scully M.O. "Modeling focusing Gaussian beams in a turbid medium with Monte Carlo simulations," Opt. Exp., 23(7), 8699-8705 (2015).

[14] Kirillin M.Y., Meglinski I., and Priezzhev A.V. "Effect of photons of different scattering orders on the formation of a signal in optical low-coherence tomography of highly scattering media," Quantum Electron., 36(3), 247-252 (2006).

[15] Churmakov D.Y., Kuzmin V.L., and Meglinski I. "Application of the vector Monte Carlo method in Polarization Optical Coherence Tomography," Quantum Electron., 36(11), 1009-1015 (2006).

[16] Kirillin, M., Meglinski, I., Sergeeva, E., Kuzmin V.L., and Myllyla R. "Simulation of optical coherence tomography images by Monte Carlo modelling based on polarization vector approach”, Opt. Express, 18(21), 21714-21724 (2010).

[17] Kandidov V.P., Militsin V.O., Bykov A.V., Priezzhev A.V. "Application of corpuscular and wave Monte-Carlo methods in optics of dispersive media," Quantum Electron., 36(11), 1003-1008 (2006).

[18] Glaser, A. K., Chen, Y., Liu, J. T. C. "Fractal propagation method enables realistic optical microscopy simulations in biological tissues," Optica 3(8), 861-869 (2016). 